\title{
La construcción social de los espacios para la memoria sobre el terrorismo de Estado en Argentina como lugares de memoria auténtica
}

\author{
Ana Guglielmucci \\ Doctora en Antropología (Universidad de Buenos Aires) \\ Investigadora del Instituto de Ciencias Antropológicas \\ mucciana@hotmail.com
}

\begin{abstract}
Resumen En la Argentina, la memoria pública de eventos ligados a la desaparición forzada de personas es evocada, entre otras formas, por medio de la creación de "espacios para la memoria sobre el terrorismo de Estado", ubicados en lugares donde funcionaron centros clandestinos de detención. A partir de un estudio etnográfico, en este artículo analizo de qué manera diversos actores (políticos, supervivientes, familiares de desaparecidos, militantes de organismos de derechos humanos y organizaciones barriales) han resuelto una serie de problemas estéticos, políticos y morales ligados a la representación del terrorismo de Estado mediante estos espacios, y a su transmisión hacia las nuevas generaciones. Especialmente, me pregunto acerca de la relación entre evidencia material e interpretación histórica o, en otras palabras, de qué manera los protagonistas de este proceso de memorialización han construido una representación particular sobre las huellas del pasado que refuerza la presentación de estos lugares como espacios de memoria auténtica.
\end{abstract}

Palabras clave: Argentina; terrorismo de Estado; memoria; representación; espacio.

\section{Introducción}

Tumerosos estudiosos sociales se han abocado a debatir sobre 1 los límites representacionales de eventos críticos $^{1}$, como genocidios étnicos y religiosos, masacres de campesinos y desapariciones masivas de personas, por parte de instituciones estatales o paraestatales (Das, 1995; Friedlander, 2007). En Europa central y Estados Unidos, las discusiones de carácter filosófico, historiográfico y estético sobre este tipo de eventos han tendido a versar acerca de la crisis de la modernidad occidental, refiriéndose especialmente al tópico del Holocausto, entre otras cuestiones (Young, 1993; Huyssen, 2002). En América Latina, en cambio, los debates han tendido a centrarse en la representación sobre el terrorismo de Estado y la violencia insurgente, destacándose el estudio sobre las disputas en torno a la construcción de memorias sobre las dictaduras militares en el Cono Sur (Jelin, 2002).

1. Pensando en contextos de devastación masiva Veena Das (1995) toma el término "critical events" para referirse a la textura emocional que producen ciertos eventos traumáticos. Das retoma la noción de "acontecimiento" de Furet para designar aquellas experiencias sociales que "instituyen una nueva modalidad de acción histórica que no estaba inscrita en el inventario de esa situación". 
En el caso de Argentina, el debate sobre cómo dar cuenta del pasado reciente ha estado acompañado, simultáneamente, por el desarrollo de una amplia movilización social y la implementación de iniciativas gubernamentales tendientes a promover la memoria sobre la última dictadura militar (1976-1983) en el espacio público ${ }^{2}$. Un tipo de iniciativa, en este sentido, se ha caracterizado por la marcación social de algunos lugares que funcionaron como centros clandestinos de detención (CCD) en tanto objetos de denuncia político-jurídica. Pues, a partir de la visibilización de estos sitios, se ha procurado señalar públicamente la impunidad de los crímenes pasados (secuestro, tortura, asesinato, desaparición, apropiación de menores) y demandar a los sucesivos gobiernos nacionales la implementación de políticas estatales tendientes a garantizar "Memoria, Verdad y Justicia".

Paralelamente a esta creciente demanda social, desde fines de la década del noventa, el Gobierno de la Ciudad Autónoma de Buenos Aires (GCABA) se comprometió con la señalización de algunos lugares que funcionaron como CCD y su (re)funcionalización como "espacios para la memoria sobre el terrorismo de Estado". Dos de ellos fueron los ex CCD identificados como "ESMA" y "Olimpo"3. Para ese entonces, en el predio de la ESMA, funcionaban varias dependencias educativas de la Armada Argentina $y$, en el predio del Olimpo, funcionaba una Planta Verificadora de Automotores (PVA) de la Policía Federal Argentina (PFA).

A partir del año 2003, el Gobierno Nacional (GN) también se comprometió con la (re)funcionalización de algunos ex CCD como espacios para la memoria. Con el objetivo de promover e implemen- tar este tipo de iniciativas conmemorativas, los jefes de ambos gobiernos (el Presidente Néstor Kirchner y el intendente Aníbal Ibarra) instituyeron órganos de gestión mixta, integrados por representantes gubernamentales y no gubernamentales. Los miembros de dichos órganos fueron designados institucionalmente como los encargados de definir qué hacer en estos lugares. Entre los integrantes no gubernamentales fueron incorporados los referentes de: organismos de DD.HH., familiares de detenidos desaparecidos, supervivientes y, en contadas ocasiones, militantes de organizaciones vecinales. Entre los integrantes gubernamentales fueron incorporados los funcionarios encargados de las áreas de: patrimonio, obras públicas, espacios verdes, gestión y participación ciudadana, y derechos humanos. El "Ente" es el órgano que se dedica a gestionar el espacio para la memoria de la ESMA; la "Comisión de Trabajo y Consenso" (CTyC) es la que se encarga del Olimpo ${ }^{4}$.

Durante más de una década, la demanda de "Memoria, Verdad y Justicia" ha sido desplegada como una cadena semántica unívoca frente al Gobierno. Sin embargo, tal hilaridad resulta vaporizada incluso para los propios actores que la levantan como consigna política, cuando ellos mismos son los encargados de definir la (re)funcionalización de los CCD como espacios para la memoria. ¿Cómo dar cuenta de lo sucedido en estos lugares?, ¿Cuál es el sentido de transmitirlo?, ¿Quiénes son sus destinatarios? Los ex CCD remiten a sucesos históricos complejos cuya comprensión incluye el establecimiento de continuidades y rupturas con la actualidad. Es decir, ellos remiten a cierta información incómoda no sólo sobre el período en el que funcionaron como CCD,

2. En este artículo nos centramos en los debates de los propios actores con respecto a la interpretación y uso dados al concepto de memoria, por sobre el desarrollo de una definición teórica. Para un análisis crítico acerca de los estudios sociales sobre memoria en Argentina pueden consultarse las obras de Rosana Guber y Sergio Visakovsky (2005) y Visacovsky (2007), quienes dan cuenta de qué manera los intelectuales argentinos han tendido, frecuentemente, a superponer las investigaciones sobre este fenómeno con el compromiso político con la realidad estudiada. En este sentido, señalan el carácter reconstructivo (en términos históricos) y moralizante (en términos políticos) de algunos de estos trabajos.

3. El CCD ESMA funcionó entre 1976 y 1983 en las dependencias de la Escuela de Mecánica de la Armada, situadas sobre la Av. Libertador, en el barrio de Núñez. Sus instalaciones ocupan aproximadamente unas 14 hectáreas. La Marina continuó ocupando el predio hasta el año 2004, cuando los representantes del Gobierno Nacional y Metropolitano firmaron un convenio que dispuso su destino como "espacio para la memoria sobre el terrorismo de Estado". A partir de la firma de este convenio comenzó un proceso de relocalización de las escuelas navales que culminó en el año 2007. El CCD Olimpo funcionó entre mediados de 1978 e inicios de 1979, en dependencias de la División de Automotores de la Policía Federal Argentina. El predio ocupa aproximadamente una hectárea, situada en el barrio de Floresta. En el año 2004, ante la fuerte movilización de organizaciones barriales, los representantes del GN y GCABA firmaron un convenio destinándolo como futuro espacio para la memoria. A mediados de 2005, las instalaciones de la Policía fueron trasladadas a otro lugar.

4. El Ente está integrado por un representante del Poder Ejecutivo Nacional (cuya representación fue delegada en la Coordinadora del Archivo Nacional de la Memoria), un representante del Poder Ejecutivo del GCABA (cuya representación fue delegada en la Secretaria Ejecutiva del Instituto Espacio para la Memoria), y un representante de un Directorio, integrado por representantes de organizaciones de DD.HH. Dicho Directorio fue conformado por quince miembros, catorce de ellos en representación de los siguientes organismos de DD.HH.: AEDD, APDH, Abuelas de Plaza de Mayo, Asociación Madres de Plaza de Mayo, Buena Memoria, CELS, Familiares de Desaparecidos y Detenidos por Razones Políticas, Fundación Memoria Histórica y Social, Herman@s, H.I.J.O.S., LADH, Madres de Plaza de Mayo - Línea Fundadora, MEDH, SERPAJ; y un representante elegido por el Consejo Asesor, integrado por ex detenidos-desaparecidos. La CTyC fue integrada, inicialmente, por un representante de la SSDH del GCABA, sobrevivientes y familiares de detenidos-desaparecidos, representantes de seis organismos de DD.HH. (Abuelas y Madres de Plaza de Mayo-Línea Fundadora, H.I.J.O.S., Familiares, AEDD, Herman@s), y dos organizaciones barriales ("Vecinos por la Memoria" y "Buscando un Destino de Uso Público para "El Olimpo"- Red GAO"). 
sino también sobre las acciones llevadas a cabo hasta el momento por parte de los sucesivos Gobiernos y distintos sectores de la sociedad civil con respecto a los crímenes pasados.

De este modo, al decidir qué hacer en cada lugar (re)funcionalizado como "espacio para la memoria de todos los argentinos" se ponen en juego un conjunto de variables. Cada experiencia de (re)funcionalización conlleva una serie de condicionamientos vinculados a, por ejemplo, quiénes participan de los respectivos órganos de gestión, qué rol cumplen las instituciones gubernamentales, las particularidades edilicias de cada lugar o el ámbito socio-urbano donde están ubicados. Considerando esta variabilidad, mi intención en este artículo consiste, en primer lugar, en dar cuenta del caleidoscopio de actividades desarrolladas por los integrantes de los órganos de gestión política encargados de definir qué hacer en los ex CCD ESMA y Olimpo. Y, en segundo lugar, me interesa analizar de qué manera ha sido construido un relato particular sobre determinados eventos críticos, incorporados en nuestra "Historia Nacional", a través de determinadas manifestaciones materiales como los espacios para la memoria.

Los integrantes de ambos órganos de gestión (el Ente y la CTyC) han centrado sus debates preliminares sobre qué hacer en los espacios ESMA y Olimpo en torno a dos amplios ejes, unidos a la articulación entre materialidad y memoria. El primero de ellos, ha girado en torno a la preservación, transformación y/o reconstrucción de los edificios, en tanto prueba material para la Justicia y documento testimonial para la Historia. Algunas preguntas en este sentido han consistido, por ejemplo, en si debían volver a construirse las celdas, o, al contrario, destacarse las huellas o marcas que denotan su existencia y las acciones llevadas a cabo para ocultarlas. El segundo eje, ha girado en torno a la construcción de un relato sobre el lugar, articulando pasado, presente $\mathrm{y}$ futuro de un modo particular. Las preguntas en este sentido han consistido, por ejemplo, en qué tipo de información sería necesaria para su elaboración, y la relación entre testimonio, interpretación y/o explicación. A continuación, realizo una descripción analítica de las definiciones que estos grupos fueron forjando sobre cada uno de estos espacios, a partir de su organización y marcación simbólica.

\section{Preservar y/o transformar: los ex CCD como prueba material para la Justicia}

Para los actores que participan de los órganos de gestión de los espacios para la memoria ESMA y
Olimpo, la (re)funcionalización de estos lugares constituye una afirmación pública de su histórica demanda de "Memoria, Verdad y Justicia". En este sentido, los integrantes del Ente y la CTyC comprenden primordialmente a estos lugares como testimonio material del terrorismo de Estado, potencialmente utilizable como evidencia en las causas judiciales abiertas - recientemente - por crímenes de lesa humanidad. De acuerdo con la conexión establecida entre la materialidad del lugar y los juicios penales, los integrantes de ambas comisiones comparten la premisa de preservar el lugar tal cual estaba cuando las FF.AA. y de seguridad fueron desalojadas y relocalizadas en otros predios. El argumento dominante consiste en afirmar que, es tan importante revelar el funcionamiento del lugar en cuanto CCD, como dar cuenta de las prácticas desarrolladas posteriormente por dichas fuerzas para ocultarlo pues, ello habría garantizado su impunidad durante los sucesivos gobiernos constitucionales. Esta postura preservacionista se ha impuesto, en general, sobre los planteamientos de reconstruir las celdas para explicitar el funcionamiento del CCD o, por el contrario, dinamitar sus restos como una forma de catarsis personal y colectiva.

La postura hegemónica, volcada hacia la preservación material del lugar, condujo a los integrantes de ambas comisiones a convocar a un equipo de especialistas con el objetivo de recuperar las huellas de los usos pasados dados al lugar, grabados en la estructura edilicia (paredes, pisos, etc.). Las agencias gubernamentales encargadas del funcionamiento de estos espacios, la Subsecretaría de DD.HH (SSDH) del GCABA y el Archivo Nacional de la Memoria (ANM) del GN, incorporaron a arquitectos, arqueólogos, antropólogos y conservacionistas, con el fin de rastrear y salvaguardar las marcas edilicias que denotan el funcionamiento pasado como CCD, y las sucesivas modificaciones realizadas con el objetivo de ocultarlo. Si bien, en el marco de la reapertura de las causas judiciales, la premisa era "preservar todo", a medida que fue avanzando el trabajo de relevamiento arqueológico y profundizándose el debate al interior de las comisiones sobre qué hacer en cada lugar, surgieron nuevas preguntas sobre qué conservar y para qué.

En un inicio, la mayoría de los integrantes de las comisiones - encargadas de definir la organización y contenido simbólico de los espacios para la memoriamanifestaron la prioridad de conservar el sector donde estaban las celdas y salas de interrogatorio o "quirófanos". Esta postura, como ya enunciamos, se impuso frente a la de aquellos grupos que planteaban: reconstruirlo tal cual era cuando funcionó como CCD, o, demolerlo completamente para darle una nueva imagen, "ligada a la vida y no a la muerte". En el caso de la ESMA, por ejemplo, la Asociación de Ex Detenidos Desaparecidos (AEDD) propuso reconstruir el sec- 
tor donde fueron recluidos los prisioneros, de acuerdo con el período específico de funcionamiento de ese CCD que se pretendiera explicar. En el caso del Olimpo, en un primer momento, los representantes de AEDD, H.I.J.O.S y algunos sobrevivientes también propusieron reconstruir las celdas derruidas con la intención de patentizar los crímenes allí cometidos. No obstante, en ambos casos, luego de varias reuniones entre representantes de DD.HH, funcionarios gubernamentales y un grupo de profesionales (conformado por arquitectos, restauradores, arqueólogos y antropólogos), los integrantes de ambos órganos de gestión política acordaron la realización de reproducciones gráficas y/o digitales que, facilitaran la comprensión del funcionamiento del campo de detención sin necesidad de realizar reconstrucciones edilicias de las celdas o réplicas de los implementos de tortura. En síntesis, en ambos casos, se consensuó un criterio conservacionista y de reconstrucción virtual de operación de los CCD (mediante maquetas, planos, infografías, animaciones tridimensionales, etc.), por sobre un criterio de reproducción material o transformación total.

Según los integrantes de los respectivos órganos de gestión, el propósito general del trabajo de preservación apunta a descubrir las marcas edilicias que evidencian el accionar del CCD y posterior ocultamiento de las fuerzas militares y policiales. Sin embargo, la historia de los edificios donde funcionaron los CCD, por medio de sus capas de pintura o modificaciones arquitectónicas, permite construir un relato propio, más amplio y heterogéneo. A partir del trabajo de los conservacionistas se obtiene una colección de marcas, acompañadas por una colección de relatos, que no siempre son coincidentes entre sí. En un inicio, los integrantes de ambas comisiones plantearon la relación entre materialidad y memoria como una relación directa, en la que la materia apoya, refuerza, y valida los recuerdos de los sobrevivientes (especialmente, sus testimonios personales sobre lo sucedido en los CCD). No obstante, a medida que fue avanzando el trabajo de los conservacionistas, la caracterización de esta relación debió ser replanteada, pues, la materialidad no funcionaba únicamente como un refuerzo de los testimonios de los sobrevivientes, sino que también permitía discutirlos, relativizarlos y/o ampliarlos. El trabajo de registro de las marcas edilicias se reveló más complejo de lo que esperaban e impuso arduos debates al interior de las respectivas comisiones. Por ejemplo, la prioridad dada a la actividad de preservación, requirió decidir si era necesario registrar y conservar la totalidad del predio y, a su vez, cuál era la profundidad temporal de las marcas edilicias que querían encontrar, y/o los testimonios que pretendían constatar por intermedio de ellas.

En el caso del Olimpo, se dio una fuerte discusión al interior de la CTyC sobre qué conservar y qué transformar. Antes de ser oficializado en cuanto espacio para la memoria, el predio fue utilizado, sucesivamente, como: terminal de tranvías, terminal de ómnibus, CCD, y Planta Verificadora de Automotores. En este sentido, los integrantes de la CTyC se preguntaban, por ejemplo, si debían remover o conservar el tinglado de la vieja estación de tranvías, elemento icónico del lugar como sitio histórico, pero difícil de mantener y de reponer. Finalmente, las decisiones consensuadas fueron ajustadas fundamentalmente a la decisión de exhibir el funcionamiento del lugar como CCD y su posterior camuflaje, sin alterar su estructura edilicia general. Es decir, si se conserva el tinglado que cubre la totalidad del predio es porque permitió a los sobrevivientes identificar el lugar desde el exterior una vez que fueron liberados; si no se reconstruye una celda es porque consideran que el crimen allí cometido se perpetúa con la impunidad alentada por su ocultamiento.

Entre aquellos actores que participan en el Ente se dio un debate similar sobre qué preservar y qué transformar respecto al espacio material. No obstante, este debate se enmarca en ciertas diferencias vinculadas a las características de cada uno de los predios y el funcionamiento pasado de cada uno de los CCD. A diferencia del CCD Olimpo, que funcionó seis meses, la ESMA funcionó aproximadamente siete años, durante los cuales fueron realizadas numerosas remodelaciones. Por ejemplo, durante la visita in loco de la Comisión Interamericana de DD.HH (CIDH), en septiembre de 1979, se hicieron una serie de reformas edilicias con el objetivo concreto de ocultar cualquier tipo de prueba que diera cuenta de que allí funcionaba un CCD (tal como habían denunciado los detenidos-desaparecidos posteriormente liberados y radicados en el extranjero). Dado que los sobrevivientes habían testimoniado haber sido bajados por una escalera que daba a un sótano (donde se encontraban las salas de tortura), los miembros de la $\mathrm{Ar}-$ mada desmontaron la escalera y la tapiaron con un piso enlosado. Cuando los restauradores comenzaron a trabajar en el lugar, hallaron una mancha de humedad que denota la inclinación y ubicación original de la escalera. En relación con ello, uno de los planteos al interior del Ente consistió en cómo mantener la marca de humedad para dar cuenta de la existencia de la escalera. Es decir, no se trata de reconstruir la situación original, sino justamente de mostrar las huellas de lo que hubo y los sucesivos intentos de ocultamiento por parte de la Marina. Sin embargo, ¿cómo transmitir al visitante no especializado en las técnicas de conservación, la importancia simbólica de una mancha de humedad en la pared? A su vez, ¿este tipo de marcas deben ser relevadas en los 36 edificios, distribuidos en las 14 hectáreas que ocupa el predio de la ESMA? ¿Son las huellas de la estructura funcional del 
CCD el único medio, o quizás lo más adecuado para transmitir a quienes no vivieron lo que allí sucedió?

Para los integrantes de ambas comisiones, las tareas de conservación material están fuertemente relacionadas con trabajos de investigación histórica y testimonial, enfocados en la obtención de "Verdad" y "Justicia" (consignas primordiales para el movimiento de DD.HH. en Argentina). Pero, a su vez, la conservación e investigación están relacionadas con las tareas de transmisión de la memoria sobre el terrorismo de Estado a las personas que no vivieron dichas experiencias (a través de la construcción y difusión de relatos sobre estos lugares). En este sentido, si bien los integrantes de ambas comisiones atribuyen a estas marcas edilicias el status de "documento material" (ya sea con carácter jurídico o histórico), su reconocimiento como tal depende de la selección de los testimonios que las acompañan; pues, quienes no vivieron los sucesos allí ocurridos necesitan - inevitablemente - de la transmisión de relatos, para poder conocerlos y, reconocer en la materialidad a la que aluden, la fuerza que los constata.

Durante las reuniones en las que participan los integrantes del Ente y la CTyC es común escuchar la afirmación: "estas paredes hablan", refiriéndose a la materialidad de los ex CCD. Esta frase, y todos los supuestos envueltos en la misma, implican un tipo particular de vínculo con la materialidad de estos espacios. Los edificios, como objetos que nos trascienden, parecen tener un poder mayor que las meras palabras. Sin embargo, siempre es por medio del lenguaje que intentamos comprender sus significaciones y que podemos transmitirlas a los otros, incluso a las generaciones venideras. La materialidad tiene un poder ambiguo. Este poder peculiar, siguiendo a Susan Pearce (1992), emana de la habilidad de los objetos de ser simultáneamente signos y símbolos, al traer una verdadera parte del pasado al presente y, a su vez, cargar eternas reinterpretaciones simbólicas. Un objeto relacionado con un evento sobrevive físicamente al mismo. Con el tiempo el material mantiene una relación metonímica con el evento del pasado, es parte intrínseca del evento. En tanto signo el objeto acarrea sentido, una eterna relación con el pasado, y esto es lo que experimentamos como el poder del objeto en sí mismo, enlazado a su aura de autenticidad.

A partir del trabajo de los integrantes del Ente y la $\mathrm{CTyC}$, las marcas edilicias sobre el funcionamiento del CCD y su ocultamiento posterior se han convertido, de algún modo, en "objetos patrimoniales". Según Krzysztof Pomian, los museos - como institución -conservan objetos puestos entre paréntesis, por lo cual más allá de ser conceptualizados como lugares de memoria también se pueden entender como "máquinas de olvido activo de una temporalidad exterior de la que se extraen los objetos expuestos" (1990, 179). Si bien los espacios para la memoria no son definidos como museo, y las marcas edilicias no son idénticas a los objetos patrimoniales coleccionados en ellos, creo que podemos hacer extensiva la reflexión de Pomian al tipo de selección operada en estos lugares, pues, con la exposición de marcas edilicias, los integrantes de las respectivas comisiones procuran retrotraer al visitante hacia lo que fue, por medio de lo que es. Con la ayuda de la exposición de una colección de marcas y unos pocos objetos hallados durante los trabajos de restauración y relevamiento arqueológico, ellos proponen a quien visita el lugar un pacto tácito, lo cual implica aceptar que la marca edilicia es una huella del pasado equivalente a su interpretación actual. Es decir, la presentación de las marcas edilicias y los objetos arqueológicos en términos de "testimonio" o "recuerdo vivido", supone al visitante aceptar el como $s i$ en términos de lo que realmente fue y ya no lo es.

Una mirada crítica sobre la representación de estos espacios en tanto lugares de memoria auténtica (a partir de dicha exposición objetual que conecta pasado y presente a través de las marcas edilicias), advierte los peligros de "saturar lo cultural con lo cultual, es decir, la cultura con el culto" (Sarlo, 2009). Beatriz Sarlo señala al respecto que, "el culto de un pasado a través de los restos materiales que subsisten en la ciudad o son reconstruidos, como si no hubieran atravesado una etapa de desaparición y obnubilación, se origina en una arqueología de los remanentes que evocan la historia, y muchas veces, más que presentarla, la sustituyen" (2009, 505). Este es otro de los desafíos que se le plantea a los integrantes de ambas comisiones, que el privilegio dado a las marcas edilicias, como testimonio auténtico del pasado, no sustituya la construcción de relatos históricos que contribuyan a otro de sus principales objetivos políticos: que la denuncia sobre los crímenes pasados ayude a "prevenir nuevas violaciones a los DD.HH. en el futuro". Con base en este tipo de preocupaciones, ellos mismos se preguntan cuál es el sentido de la memoria, y si estos espacios se limitan a funcionar - primordialmente - como prueba material para la Justicia. De acuerdo con las respuestas que han ido construyendo colectivamente, los integrantes de las respectivas comisiones fueron plasmando distintos relatos sobre la materialidad de dichos lugares.

\section{La categorización y sectorización simbólica de los espacios para la memoria}

Para los actores que han participado en el proceso de marcación social y gestión de los espacios para la memoria, uno de los ejes de debate iniciales ha gi- 
rado en torno a si las FF.AA y de seguridad debían ser desalojadas en su totalidad de los respectivos predios. En su mayoría, los integrantes de ambas comisiones definieron que no aceptarían la "convivencia" con miembros de las fuerzas que habían sido responsables por los crímenes de lesa humanidad allí cometidos. En el caso de la ESMA, el Centro de Estudios Legales y Sociales (CELS) fue una de las pocas organizaciones que hizo una propuesta diferente. En primer lugar, planteó que parte de los liceos navales continuaran funcionando en el lugar. Y, en segundo lugar, manifestó que sus estudiantes asistieran a las futuras actividades de formación en DD.HH. En el caso del Olimpo, la posibilidad de que parte del predio continuara en manos de la PFA no fue considerada por ninguna de las organizaciones que integran la CTyC. Este debate se produjo, en cambio, con algunos vecinos del lugar (que no integran la Comisión). Estos últimos reclamaron que se conservara apenas un sector del predio como "sitio de memoria", y que se dejara el resto para la permanencia de la PFA, o para el desarrollo de otro tipo de actividades, no asociadas directamente al repudio del terrorismo de Estado, sino, más bien a las necesidades socioeconómicas de los habitantes del barrio (como talleres de oficios, oficinas de asesoría legal, bolsas de trabajo, etc.).

Tras determinar que las FF.AA y de seguridad debían desocupar cada uno de los respectivos predios en su totalidad, el principal eje de debate giró acerca de si todo el lugar debía ser utilizado para "hacer memoria sobre el terrorismo de Estado", o sólo el sector donde permanecieron recluidos los detenidosdesaparecidos (denominado "Casino de oficiales", en el caso del CCD ESMA, y "Pozo", en el caso del CCD Olimpo). Dos posiciones enfrentadas se demarcaron en este sentido, las que fueron desentrañadas de un modo particular, según cada una de las comisiones. Una de las posiciones minoritarias, sostenida con fuerza por la AEDD, se opuso a cualquier otro tipo de uso del espacio que no se orientara a dar cuenta del genocidio allí perpetrado. Mientras que la otra posición, que resultó ser la mayoritaria, propuso destinar sólo la parte donde fueron recluidos los detenidosdesaparecidos a este objetivo, dejando el resto del predio para otros usos posibles, ligados a actividades culturales, administrativas, investigativas e informativas.

Con relación a los posibles usos del espacio, la AEDD indicó que su fragmentación funcional expresaría una visión equivocada sobre el terrorismo de Estado y la lógica interna de los CCD como "maquinarias represivas". En primer lugar, porque todos los integrantes de las FF.AA y de seguridad que trabajaron allí en ese momento habrían sido "cómplices" (ya sea por acción, coacción u omisión). Y, en segundo lugar, porque todas sus dependencias habrían permitido asegurar la reproducción material del CCD o encubrir su funcionamiento. En el caso de la ESMA, la AEDD se opuso rotundamente a que el lugar fuera utilizado con otra finalidad que no fuera la de denunciar su funcionamiento como CCD. En este sentido, se opuso a la instalación de oficinas públicas, refiriéndose explícitamente al ANM y al Instituto Espacio para la Memoria (IEM). Esta decisión fue fundamentada en que el movimiento generado por la cotidianeidad de estas dependencias gubernamentales vaciaría de contenido simbólico al lugar. En sus propias palabras, porque "donde hubo muerte debe señalarse, recordarse, mostrarse, saberse que hubo muerte, quiénes fueron los que murieron, por qué murieron y quiénes los mataron. No debe pretenderse que ahora haya vida" . De hecho, cuando fue creado el Ente, la AEDD fue uno de los pocos organismos de DD.HH que no solicitó ningún edificio dentro del predio de la ESMA. Más allá de que algunos militantes de DD.HH., sobrevivientes e, incluso, representantes gubernamentales, reconocieron la potencia simbólica del "vacío" y la ausencia de rutinas que tiendan a la naturalización del funcionamiento cotidiano del lugar, postularon que ello era difícil de alcanzar y sostener. Entre otros motivos, alegaron que ello implicaba dejar vacante un predio de 14 hectáreas con 36 edificios en medio de una ciudad con una fuerte demanda habitacional.

La mayoría de las agencias estatales y organizaciones no gubernamentales que componen el Ente y la CTyC plantearon un posicionamiento diferente a la AEDD. Gran parte de sus integrantes argumentaron que si bien podía considerarse que la totalidad del predio de la ESMA y el Olimpo fueron adecuados a su funcionamiento como CCD, ello no implicaba necesariamente dejarlo tal cual estaba o reconstruirlo tal cual era anteriormente. Entre otras razones, arguyeron que más allá de su preservación como testimonio material para la Justicia, la (re)funcionalización como espacio para la memoria implicaba desarrollar ciertos vehículos de transmisión para que los que no vivieron esa experiencia pudieran comprenderla. Es decir, a la preocupación sobre qué preservar, se sumó la preocupación sobre cómo explicar lo sucedido a las nuevas generaciones.

Luego de los primeros dos años de trabajo conjunto, los integrantes de ambas comisiones llegaron a tres grandes consensos para definir los contornos de los espacios para la memoria. El primer ha sido no coexistir con las FF.AA. y de seguridad en el mismo predio. El segundo ha sido preservar el lugar tal cual 
estaba al ser desalojado. Y, el tercero, ha consistido en marcar todo el lugar externamente como ex CCD, y no apenas la parte donde estuvieron recluidos los detenidos-desaparecidos. La conceptualización de los predios como totalidad, en cuanto espacio para la memoria, enmarcó todos los demás debates a futuro sobre qué conservar o no en cada uno de ellos, qué narraciones construir sobre lo allí acontecido, y cómo imprimirlo simbólicamente en el espacio físico. Sin embargo, no todos acordaban sobre qué se podía hacer en su interior.

La AEDD, como ya mencionamos, insistió en que el predio (como objeto de resguardo material por su carácter probatorio en las causas judiciales) no debía tener otro destino ni función que el de ser "testimonio material del genocidio". Esta postura implicaba reconstruir el lugar tal cual era cuando funcionaba como CCD, con el objetivo de mostrar el accionar de las FF.AA. y de seguridad, además de representar la identidad de los detenidos desaparecidos allí secuestrados. A diferencia de la AEDD, las demás organizaciones de DD.HH. plantearon la alternativa de distinguir entre el rol testimonial del lugar como "sitio histórico" (reservado para el sector donde fueron recluidos los detenidos-desaparecidos), del rol explicativo sobre el terrorismo de Estado, sus antecedentes y consecuencias (localizado en otro sector en el interior del predio).

Con base en esta última propuesta, la mayoría de los integrantes de ambas comisiones tendieron a diferenciar las actividades desarrolladas en cada una de estas áreas, tanto material como simbólicamente. En el sector diferenciado como "testimonio material sobre el terrorismo de Estado", privilegiaron las tareas de conservación, relevamiento de marcas edilicias y, señalización sobre el funcionamiento del lugar como CCD y las sucesivas modificaciones hechas para ocultarlo. En el otro sector, en cambio, los consensos fueron mucho más ambiguos. En algunos casos, decidieron conservar los edificios tal cual estaban; en otros casos, decidieron remodelarlos o, excepcionalmente, desmantelarlos y crear nuevas estructuras, como un anfiteatro o una plaza pública.

En el caso de la ESMA, los integrantes del Ente (a excepción de la AEDD) deslindaron la utilización de algunas áreas para dedicarlas a la "memoria sobre el terrorismo de Estado", y sugirieron otro tipo de uso para el resto del predio. Con relación a estos otros usos posibles, los condicionaron al carácter público de los emprendimientos y a su vinculación con la defensa y promoción de los DD.HH en la actualidad. Algunas propuestas incluyeron espacios para acciones sociales dirigidas a los sectores más vulnerados en sus derechos y perjudicados económicamente. Sin embargo, se terminó imponiendo su utilización para actividades culturales y recreativas, y la instalación de dependencias gubernamentales encargadas de "preservar la memoria" y "promover los DD.HH.".

Con respecto al sector dedicado a la "memoria sobre el terrorismo de Estado", a su vez, los integrantes del órgano de gestión política de la ESMA distinguieron entre: un área para ocuparse de la "construcción de la memoria" y un área definida como "sitio histórico". Una de las primeras actividades desarrolladas por el Ente, en lo que sus propios integrantes definen como "sitio histórico", consistió en diseñar una serie de carteles explicativos sobre el funcionamiento del lugar como CCD. Los carteles, instalados en junio de 2005, fueron ubicados desde el lugar de ingreso al predio hasta el Casino de Oficiales, siguiendo el recorrido al que eran sometidos los detenidos-desaparecidos. Ellos fueron confeccionados por técnicos de la SSDH, junto a un equipo asesor de profesionales, sobrevivientes, familiares de detenidos-desaparecidos y militantes de DD.HH. En el diseño de los carteles incluyeron planos, croquis, textos explicativos y fragmentos de los testimonios de los sobrevivientes, con la intención de asistir a la comprensión y contextualización del funcionamiento del CCD. De acuerdo con el Informe de la Comisión Bipartita, realizado en el mes de marzo del año 2007, ellos constituyen "la única intervención a realizarse en el Casino, que será mantenido sin modificación ni reconstrucción alguna". Sin embargo, como ya esbozamos anteriormente, la preservación de las marcas edilicias es un tipo de intervención específica en este sector. Pues, ellas son utilizadas para revalidar la información volcada en la señalización, sustentada en las declaraciones de los sobrevivientes.

El relevamiento y preservación material de las marcas edilicias, a pesar de ser altamente valorada por los integrantes de ambas comisiones, es invisibilizada por ellos como un tipo de intervención. Esta invisibilización se explica, de algún modo, porque esta forma de intervención constituye un recurso medular en la legitimación (material y simbólica) de los relatos construidos sobre el espacio (a lo que anteriormente nos hemos referido como el aura de autenticidad). De hecho, la instalación de cada uno de los carteles, más allá de la definición de su contenido, implicó arduos debates al interior de cada una de las comisiones. Por ejemplo, algunas de las discusiones giraron sobre cuál era la manera adecuada de hacerlo para no transformar radicalmente el lugar y dejar marcas irreversibles en el "sitio histórico". Sin duda, la colocación de estos carteles transformó el lugar, tanto en términos materiales como simbólicos, ajustándose al cambio de destino en tanto espacio para la memoria. Resulta significativo, no obstante, el trabajo dedicado por las comisiones para minimizar este tipo de intervención, presentándola como si por intermedio de ella el lugar no hubiese sido transfigurado. Así, es común la apela- 
ción de sus integrantes al "peligro" de alterar el lugar como sitio histórico, equiparándolo con un "daño" irreparable. Quizá, esto sea así porque ello afecta justamente - su aura, como lugar de memoria auténtica.

En el caso del Olimpo, los integrantes de la CTyC (en sintonía con la decisión general de conservar el lugar y no reconstruirlo tal cual era cuando funcionaba como CCD) trataron de no transformarlo irreversiblemente, preservando el sector del Pozo. Esta postura implicó no volver a levantar la parte - ahora derruida - del muro prefabricado de cemento, construido en la época de la Dictadura para incomunicar a los detenidos-desaparecidos en un sector del predio. Con el asesoramiento de un grupo de arquitectos, decidieron colocar una estructura desmontable de hierro y vidrio en la apertura del muro, diseñada de tal manera que permitiera percibir el interior y no generara sensación de encierro. La estructura operaría a modo de acceso al área del Pozo, produciendo una bisagra entre los dos sectores del predio (áreas "sitio histórico" y "construcción de la memoria"), y serviría como soporte de comunicación gráfica sobre el funcionamiento del CCD. En el mensaje de entrada al Pozo, los integrantes de la CTyC decidieron remarcar la existencia de un plan sistemático de exterminio a nivel nacional, y el funcionamiento del Olimpo como CCD en particular. En el mensaje de salida, en cambio, procuraron apelar a la sensibilidad del visitante con el objetivo de predisponerlo a comprometerse con las actividades desarrolladas actualmente en el lugar (ya sea por medio de la evocación de las personas desaparecidas y la promoción de una transformación política en el presente). De esta forma, la estructura de acceso al Pozo funcionó a modo de umbral (material y simbólico), entre un sector catalogado como "sitio histórico" (el que hace referencia exclusivamente al funcionamiento del CCD) y, otro sector, donde se realizan actividades destinadas a articular pasado y presente en un sentido que sobrepasa la preservación de la memoria sobre el terrorismo de Estado.

Paralelamente al debate sobre qué medidas adoptar para garantizar la preservación del lugar, los integrantes de la CTyC consideraron la necesidad de impulsar la investigación sobre lo sucedido en el CCD Olimpo para distinguir la relevancia de la materialidad a preservar y facilitar el trabajo de los conservacionistas a la hora de interpretar las marcas edilicias encontradas. En un primer momento, trabajadores de la SSDH, junto a los sobrevivientes del lugar, comenzaron a recabar y sistematizar información sobre las personas allí detenidas-desaparecidas, los niños nacidos durante el cautiverio de sus madres, la identidad y jerarquía de los represores (algunos de los cuales sólo son conocidos por sus apodos), el funcionamiento interno del Olimpo y su conexión con otros CCD.
El resultado de esta investigación, por un lado, les permitió diseñar las visitas guiadas, brindar información a familiares, amigos y/o compañeros de militancia de las personas allí detenidas-desaparecidas, y aportar nuevos datos a las causas judiciales. Pero, por otro lado, condujo a un debate sobre qué hacer con la información obtenida, dónde y cómo exponerla al interior del propio predio.

Por ejemplo, los integrantes de la CTyC decidieron confeccionar un listado con los nombres de los detenidos-desaparecidos y asesinados, y una bandera con sus fotos, lo que derivó en el problema de la localización de las exposiciones referidas a ellos. En un principio, decidieron que en el Pozo solamente se haría referencia al funcionamiento del CCD, la identidad de los represores, y las causas y consecuencias del terrorismo de Estado; evitando volver a colocar a los desaparecidos nuevamente en un "espacio de muerte" junto a sus represores. Para rememorar a los desaparecidos, entonces, proyectaron construir un "espacio de vida", donde se pudiera recuperar sus valores y evocar su presencia en la actualidad. De este modo, más allá de la conceptualización integral del espacio como ex CCD, comenzó a definirse una sectorización bipolar del predio, entre un "espacio de muerte" - "sector de escrache a los responsables del terrorismo de Estado", y un "espacio de vida" - "sector de homenaje a los detenidos-desaparecidos"; entre un sector a ser preservado como "testimonio material del genocidio" y un sector a ser transformado como "lugar de usos múltiples" (salas de reunión y exposiciones artísticas, oficinas administrativas, biblioteca, archivo, anfiteatro, etc.).

Los sobrevivientes del CCD Olimpo fueron unos de los pocos actores que relativizaron esta distinción, y se encargaron de que fuera readecuada de acuerdo con sus observaciones. Algunos de ellos indicaron que en el Pozo no apenas hubo sumisión, sino también resistencia. De hecho, la mayoría de los sobrevivientes relata la vida en el CCD en términos ambiguos, describiéndolo como un "lugar de muerte", pero también de "vida"; un lugar de "dolor y alegría"; de "llanto y risa"; de "coacción y aguante", de "delación y compañerismo", de "traiciones y lealtades". A partir de los testimonios de los sobrevivientes y sus observaciones posteriores, por lo tanto, surgieron nuevas inquietudes sobre cómo narrar las experiencias pasadas dentro del campo, donde los hechos no pueden ser relatados en términos morales polarizados, del tipo "buenos" y "malos".

Un tema controvertido entre los sobrevivientes y los familiares de los detenidos-desaparecidos ha girado en torno a la supervivencia de algunos y no de otros, y el "fantasma de la colaboración con el enemigo" (sospecha que, no sólo hace referencia a la delación bajo tortura y al trabajo administrativo 
dentro del campo, sino también a la participación en la aplicación de tormentos de unos detenidos sobre los otros). Si bien para los integrantes del Ente y la CTyC, la posición explícita respecto a la colaboración de los detenidos-desaparecidos es que ella fue parte de la estrategia de los captores para aniquilarlos y desarticular los lazos de confianza entre compañeros; para quienes convivieron ahí dentro, las experiencias no son fácilmente conceptualizadas en términos binarios, del tipo "blanco" o "negro".

En el caso de la ESMA, la convivencia y la colaboración con los marinos dentro del CCD fueron parte de un tema que se hizo público mediante los testimonios recogidos y divulgados en el informe Nunca Más, elaborado por la CONADEP (1984). Los propios sobrevivientes describieron y clasificaron la colaboración de los detenidos dentro del campo, con la realización de distintas tareas administrativas y de propaganda política para el proyecto de poder del Almirante Massera, en una gradiente que iba desde la esclavitud hasta la colaboración activa. Esta clasificación, sin embargo, también se caracteriza por su ambigüedad, pues uno de los mecanismos de los detenidos fue simular la cooperación con sus captores. En su obra Poder y desaparición: los campos de concentración en Argentina (1998), Pilar Calveiro, sobreviviente del CCD ESMA, se ha ocupado de describir los alcances del "poder concentracionario y desaparecedor" y sus consecuencias subjetivas actuales sobre la sociedad en general, renunciando a la apelación a una lógica binaria, que divide la experiencia de los campos en la de "héroes" y "traidores", o que identifica a la sociedad como "inocente" o "cómplice". Respecto a la vida al interior de los campos, explica que este tipo de argumentos son injustos e insuficientes, pues, los detenidos-desaparecidos simultáneamente pudieron resistir, someterse, confrontarse. En este sentido, convoca a rescatar lo que ella llama las "virtudes cotidianas" dentro de los campos, en vez de retratar la experiencia de los desaparecidos como la de una masa inerme en manos de un poder absoluto. Si bien esta convocatoria ha sido explicitada por Calveiro en numerosas actividades organizadas por militantes de organizaciones de DD.HH y políticos, en el caso del espacio para la memoria ESMA, la referencia a las "virtudes cotidianas" no ha sido incorporada como un elemento significativo en la señalización sobre el funcionamiento interno del CCD.

En el caso del Olimpo, en cambio, el tema de la "colaboración con el enemigo" no fue un tema que tomó estado público, mas bien este tema es conversado de modo privado entre algunos sobrevivientes. En especial, este tema de conversación se ha reactivado a partir de la reapertura de los juicios y la estrategia defensiva implementada por los abogados de los represores, quienes han intentando reflotar la colaboración de los propios detenidos en la aplicación de tormentos, con el objetivo de diluir la responsabilidad de los captores y general desconcierto entre la audiencia. Los sobrevivientes que participan activamente de la CTyC, han señalado dos cuestiones en este sentido: en primer lugar, no hacer referencia pública, en la medida de lo posible, a situaciones puntuales controvertidas por parte de algunos detenidos-desaparecidos, tanto en los juicios como en las visitas guiadas. $\mathrm{Y}$, en segundo lugar, describir el CCD no sólo en términos de un "espacio de muerte", pues ello no da cuenta de la totalidad de la experiencia vivida por los detenidos, en tanto no representa las manifestaciones de dignidad y resistencia personal creadas y sostenidas a pesar del sometimiento y la denigración personal constante por parte de los represores. Además, podemos agregar, si los campos son definidos únicamente como "espacios de muerte", ¿cómo explicar la supervivencia? En este sentido, para los sobrevivientes del CCD Olimpo, la incorporación de anécdotas sobre la vida cotidiana y la solidaridad dentro del campo han constituido un tema central en el desarrollo de la señalización del sector del Pozo, pues ellas dotan de sentido su propia experiencia como detenidos-desaparecidos liberados.

En términos generales, los integrantes de ambas comisiones tendieron a clasificar los espacios para la memoria de un modo semejante. En primer lugar, lo definieron como un todo orgánico, pero a su interior diferenciaron dos grandes sectores: una área "sitio histórico" y un área "memoria”. Un "área de conservación” y un "área dinámica”. En el área "sitio histórico", a su vez, distinguieron entre secciones con un rol testimonial (donde se ubican las marcas edilicias y los testimonios de los sobrevivientes que las acompañan) y secciones con un rol explicativo sobre los testimonios narrativos y materiales. Dos concepciones de memoria parecen convivir, de esta forma, en un mismo espacio conmemorativo. Una de ellas, está orientada a la reconstrucción histórica de los hechos, y es presentada por vía de la materialidad que trasciende a sus protagonistas. La otra, está relacionada con la creación de nuevos relatos sobre el pasado a partir del presente $y$, es trabajada mediante de la promoción de actividades político-culturales orientadas hacia una transformación social pedagógica en el futuro.

Esta clasificación del espacio se expresó también en una clasificación de las actitudes corporales adecuadas en cada uno de ellos. Dentro del área de conservación se procura que el movimiento de las personas sea pausado y silencioso, en señal de respeto a "los que faltan". Dentro del área "memoria", en cambio, se realizan actividades culturales (conciertos, obras de teatro, muestras de arte, etc.). Por otra parte, el área de conservación está despojada de todo ornamento, incluso iluminación potente. Mientras que la otra 
está iluminada y amueblada según los distintos usos culturales, educativos y administrativos.

De esta manera, los integrantes de ambas comisiones han subdividido el lugar en dos grandes sectores complementarios entre sí, que se asemejan a la distinción social entre lo "sagrado" y lo "profano", entre los que median ciertos tabúes para mantenerlos separados ${ }^{6}$. Significativamente, el sector clasificado como "sitio histórico" es consagrado como un espacio aparte del resto del predio, un área retirada de las demás actividades mundanas realizadas en el lugar. Su construcción como un sitio apartado, y sometido a ciertos tabúes respecto al comportamiento adecuado para transitarlo, opera como un elemento clave en el mantenimiento de su aura como lugar de memoria auténtica. Como si de esta manera el espacio pudiera dar cuenta de esa realidad pasada que ya no es, pero sigue siendo: el pasado no pasado y la conversión de la ausencia en presencia. La sacralización del espacio, manifestada en su ambivalencia, entre lo que es y lo que no es, entre lo clasificado y lo inclasificable, entre lo "sagrado" y lo "profano" es una manera de retener lo que ha sido, como algo que continúa viviendo en nosotros, y a través de los lugares que nos trascienden.

Los integrantes de ambas comisiones han operado diferentes modelizaciones materiales y simbólicas sobre el espacio que gestionan. Modelización que ha implicado la selección y manejo de diferentes argumentos históricos para dar cuenta de la ESMA y el Olimpo como espacios para la memoria sobre el terrorismo de Estado. Sin duda, esta selección conlleva a ciertos olvidos. De hecho, los lugares que los gestores de estos espacios consideran como "faros de la memoria", para otros son "lugares de amnesia" o de "media memoria". En este sentido, las organizaciones aunadas a las FF.AA. reclaman, por ejemplo, la incorporación de relatos sobre los secuestros y los atentados de las organizaciones revolucionarias. No obstante, si bien se debate sobre su contenido, raramente se discute el estatus de estos lugares en tanto espacios para la memoria. Pues, de algún modo, la materialidad inerte de estos espacios permite remontarse hacia experiencias pasadas (el secuestro, la tortura, la desaparición de los cuerpos) que aún hoy alcanzan a perturbarnos.

De un modo similar a lo que señala Maurice Godelier (1998) con referencia a los objetos sagrados, estos espacios-objeto condensan el poder de nombrar el pasado y crear relaciones sociales en el presente ${ }^{7}$. Poseen ese poder y lo contienen. Por eso, quienes los poseen, tienen el poder de inscribir historia pública, distribuyendo los efectos de ese poder en la sociedad (poder que está ligado sine qua non al objeto sagrado del cual emana). De ahí que estos espacios sean objeto de disputa, conquistados y conquistables. Pues quienes detentan su gestión controlan el poder de nombrar esa realidad que fue y aún es; el poder de articular pasado, presente y futuro por medio del espacio material. Para quienes no consideran estos espacios como lugares de memoria auténtica, ellos operan únicamente a modo de símbolo. Pero, para quienes comparten la creencia en el poder de estos espacios, ellos no son signo ni símbolo, ellos son cosas que poseen espíritu $\mathrm{y}$, por lo tanto, son poderosos en sí mismos ${ }^{8}$. Con ello, se realiza la sintesis entre lo decible y lo indecible, entre lo representable y lo irrepresentable. Pues, a la hora de dar cuenta de ese país foráneo que es el pasado, se procura acceder a él a partir de sus huellas, o, en otras palabras, la presencia de la ausencia.

6. El tabú es definido como una prohibición sancionada ritualmente contra el contacto con una cosa, una persona o una actividad. El objeto del tabú puede verse como sagrado o como contaminado, y la violación del mismo no es simplemente un crimen sino un acto de profanación. El tabú se asocia de manera especial con las personas que ocupan una posición liminal (en este caso, los desaparecidos). Emile Durkheim (1992) analizó el tabú como una forma de mantener la relación de oposición entre las esferas de lo sagrado y lo profano, afirmando que las reglas de separación son las marcas distintivas de lo sagrado, el polo opuesto de lo profano. Radcliffe-Brown (1974) sostuvo que es un mecanismo para destacar la importancia social de ciertas personas y objetos. Mary Douglas (2007) adoptó, en cambio, un enfoque simbólico, sugiriendo que los objetos y las personas se vuelven tabú cuando no encajan en el sistema de clasificación simbólica de su cultura. En cierto modo, este tipo de clasificaciones son aplicadas a los ex CCD, (re)funcionalizados como espacios para la memoria.

7. Maurice Godelier afirma que con los objetos sagrados "todo sucede como si los hombres no fueran los que dan un sentido a las cosas, como si las cosas, cuyo sentido tiene su origen más allá del mundo de los hombres, lo transmitieran a los hombres bajo ciertas condiciones. En otras palabras, la sintesis de lo decible y de lo indecible, de lo representable y de lo irrepresentable, se realiza en un objeto, exterior al hombre, pero que ejerce sobre los hombres, sobre su conducta, sobre su existencia, la mayor influencia" (1998, p. 197). Si bien esta definición no se ajusta totalmente a los espacios-objeto sobre los que trata este artículo (pues su origen no se explica por fuera del mundo de los hombres), ellos comparten varias de estas características.

8. Maurice Godelier entiende que "los objetos sagrados producen la síntesis, de lo real y de lo imaginario, que son los componentes del ser social del hombre. Por ello, tales objetos adquieren el mayor valor simbólico para los miembros de la sociedad que ha elaborado su código, aunque en ningún caso se vean reducidos a ser símbolos puros o meros objetos" (1998, p. 197). 


\title{
Bibliografía
}

CALVEIRO, Pilar. Poder y desaparición: los campos de concentración en Argentina. Buenos Aires: Colihue, 1998. DAS, Veena. Critical events: an anthropological perspective on contemporary India. Nova Delhi: Oxford University Press, 1995.

DOUGLAS, Mary. Pureza y peligro: un análisis de los conceptos de contaminación y tabú. Buenos Aires: Nueva Visión, 2007.

DURKHEIM, Emile. Las formas elementales de la vida religiosa. Madrid: Akal, 1992.

FRIEDLANDER, Saúl (Comp.). En torno a los límites de la representación: el nazismo y la solución final. Bernal: Universidad Nacional de Quilmes, 2007.

GODELIER, Maurice. El enigma del don. Barcelona: Paidos, 1998.

GUBER, Rosana; VISACOVSKY, Sergio. ¿Crisis o transición? Caracterizaciones intelectuales. Del dualismo argentino en la apertura democrática. Anuario de Estudios Americanos, Sevilla, v. 62, n. 1, p. 55-85, ene./jun. 2005.

HUYSSEN, Andreas. En busca del futuro perdido: cultura y memoria en tiempos de globalización. México: Fondo de Cultura Económica, 2002.
JELIN, Elizabeth. Los trabajos de la memoria. Madrid: Siglo XXI, 2002.

PEARCE, Susan. Museums, objects and collections. Leicester, UK: Leicester University Press, 1992.

POMIAN, Krzysztof. Musée et patrimoine. In: JEUDY, Henry Pierre (Ed.). Patrimoines en folie. París: Éd. de la Maison des Sciences de l'homme, 1990.

RADCLIFFE-BROWN, Alfred R. Estructura y función en la sociedad primitiva. Barcelona: Península, 1974.

SARLO, Beatriz. Vocación de memoria: ciudad y museo. In: VINYES, Ricard (Ed.). El Estado y la memoria: gobiernos y ciudadanos frente a los traumas de la historia. Buenos Aires: Del Nuevo Extremo, RBA, 2009.

VISACOVSKY, Sergio. Cuando las sociedades conciben el pasado como "memoria": un análisis sobre la verdad histórica, justicia y prácticas sociales de narración a partir de un caso argentino. Antípoda: Revista de Antropología y Arqueología, Bogotá: Universidad de los Andes, n. 4, p. 4974, ene./jun. 2007.

YOUNG, James E. Écrire le monument: site, memoire, critique. Annales ESC, n. 3, mayo/jun. 1993.

\section{A construção social dos espaços dedicados à memória do terrorismo de Estado na Argentina como lugares de memória autêntica}

\begin{abstract}
Resumo
A memória pública de eventos associados ao desaparecimento compulsivo de pessoas na Argentina é evocada mediante a criação de "espaços para memória do terrorismo do Estado". Esses espaços se localizam em estabelecimentos que, durante a última ditadura militar, funcionaram como centros clandestinos de detenção de pessoas. Por intermédio de um estudo etnográfico, neste artigo busco analisar as formas em que diversos atores (políticos, sobreviventes, parentes de desaparecidos, militantes de organizações de direitos humanos e de organizações de moradores) resolveram um conjunto de problemas estéticos, políticos e morais, associados tanto à representação do terrorismo do Estado envolvida nesses espaços quanto à transmissão às novas gerações. Em particular, interrogo a relação entre evidência material e interpretação histórica, isto é: os modos como os protagonistas desse processo de memorialização constroem uma representação particular sobre os rastros do passado, e como essa representação reforça a apresentação desses estabelecimentos como espaços de memória autêntica.
\end{abstract}

Palavras-chave: Argentina; terrorismo de Estado; memória; representação; espaço. 


\title{
The social construction of the spaces of memory on the State terrorism in Argentina as places of authentic memory
}

\begin{abstract}
In Argentina, public memory of events related to the forced disappearance of persons is evoked, amongst other possibilities, through the creation of spaces of memory dealing with State terrorism, spaces located in places that were formerly used as clandestine detention centers. Based on an ethnographic study, this article analyses how different actors (politicians, survivors, family members of desaparecidos, human rights activists and neighborhood associations) have identified and addressed a series of aesthetic, political and moral issues related to the representation of state terrorism within these spaces, as well as its transmission to younger generations. I am concerned with the relationship between material evidence and historical interpretation and, more precisely, how those involved in the process of memorialization construct a particular representation that reinforces the presentation of these places as spaces of authentic memory.
\end{abstract}

Keywords: Argentina; State terrorism; memory; representation; space.

Data de recebimento do artigo: 10/05/2011

Data de aprovação do artigo: 29/07/2011 